\title{
Ireland's Participation in the 48th International Mathematical Olympiad
}

\author{
JIM CRUICKSHANK
}

\section{Team Selection and Preparation}

The 48th International Mathematical Olympiad (IMO) took place in Vietnam from the 19-31 July, 2007. Ireland has been a participant in the IMO since 1989 and, as usual, this year a team of six second level students was selected to represent Ireland at this prestigious mathematical event. These students were selected by means of their performance in the Irish Mathematical Olympiad which took place on May 12. In that competition, the top six students were (in order)

1. Stephen Dolan (Coláiste Cholmcille, Ballyshannon, Co. Donegal).

2. Galin Ganchev (Castletroy College, Limerick).

3. Derek O'Brien (Wesley College, Dublin 16).

4. Jamie Judd (St. Gerard's School, Thornhill Road, Bray).

5. Yuecong Wang (Mount Anville Secondary School, Dublin 14).

6. Kate McCutcheon (Mount Mercy College, Model Farm Road, Cork).

As the best student in the Irish Mathematical Olympiad, Stephen Dolan was awarded the Fergus Gaines trophy. These six students would travel to Vietnam, accompanied by team leader Dr. James Cruickshank (NUIG), deputy leader Dr. Gordon Lessells (UL) and observer Dr. Mark Dukes (UCD).

The team convened, as has become customary, for a pre IMO training camp in UL in the week before they travelled to Vietnam. Special recognition must go to Gordon Lessells, who once again did a superb job of organising this training camp. 


\section{Jury Meetings and Problem Selection}

The IMO jury consists of all of the team leaders and is the primary decision making body for all IMO related matters. The jury convened on July 20, with the purpose of selecting the six contest problems from a shortlist of thirty. The Irish observer was also present at the jury meetings, although observers are not permitted to vote on any of the motions put before the jury.

It is normal for the jury meetings to take place at a venue that is far removed from the actual contest venue. This year the jury meetings took place in the spectacular setting of Halong Bay (a three hour bus journey from Hanoi). Due to Vietnamese government regulations, IMO participants were initially not permitted to leave the hotel during the pre contest jury meetings, unless accompanied by a military escort. This led to some frustration among the leaders and observers and following several appeals to the organisers, these restrictions were relaxed after a couple of days.

The selection process (long established by tradition) is usually a long (and some might say torturous) discussion of the various shortlisted problems. As usual there were a large number of exceptionally difficult problems, and not quite so many of the easy variety. Easy is of course a relative term in this context, as even the easiest IMO problem is far beyond the capabilities of a typical Irish second level student.

After two days of jury meetings the following six problems were chosen.

\section{First Day}

Problem 1. Real numbers $a_{1}, a_{2}, \ldots, a_{n}$ are given. For each $i$ $(1 \leq i \leq n)$ define

$$
d_{i}=\max \left\{a_{j}: 1 \leq j \leq i\right\}-\min \left\{a_{j}: 1 \leq j \leq n\right\}
$$

and let

$$
d=\max \left\{d_{i}: 1 \leq i \leq n\right\} .
$$

(a) Prove that, for any real numbers $x_{1} \leq x_{2} \leq \cdots \leq x_{n}$,

$$
\max \left\{\left|x_{i}-a_{i}\right|: 1 \leq i \leq n\right\} \geq \frac{d}{2} .
$$

(b) Show that there are real numbers $x_{1} \leq x_{2} \leq \cdots \leq x_{n}$ such that equality holds in (1). 
Problem 2. Consider five points $A, B, C, D$ and $E$ such that $A B C D$ is a parallelogram and $B C E D$ is a cyclic quadrilateral. Let $l$ be a line passing through $A$. Suppose that $l$ intersects the interior of the segment $D C$ at $F$ and intersects line $B C$ at $G$. Suppose also that $E F=E G=E C$. Prove that $l$ is the bisector of angle $D A B$.

Problem 3. In a mathematical competition some competitors are friends. Friendship is always mutual. Call a group of competitors a clique if each two of them are friends. (In particular, any group of fewer than two competitors is a clique.) The number of members of a clique is called its size. Given that, in this competition, the largest size of a clique is even, prove that the competitors can be arranged in two rooms such that the largest size of a clique contained in one room is the same as the largest size of a clique contained in the other room.

\section{Second Day}

Problem 4. In triangle $A B C$ the bisector of angle $B C A$ intersects the circumcircle again at $R$, the perpendicular bisector of $B C$ at $P$, and the perpendicular bisector of $A C$ at $Q$. The midpoint of $B C$ is $K$ and the midpoint of $A C$ is $L$. Prove that the triangles $R P K$ and $R Q L$ have the same area.

Problem 5. Let $a$ and $b$ be positive integers. Show that if $4 a b-1$ divides $\left(4 a^{2}-1\right)^{2}$, then $a=b$.

Problem 6. Let $n$ be a positive integer. Consider

$$
S=\{(x, y, z): x, y, z \in\{0,1, \ldots, n\}, x+y+z>0\}
$$

as a set of $(n+1)^{3}-1$ points in three dimensional space. Determine the smallest possible number of planes, the union of which contains $S$ but does not contain $(0,0,0)$.

The contestants had 4.5 hours to complete each paper. It is worth noting that many of the jury expressed concerns over the extreme difficulty of Problem 6 . One very respected jury member expressed his belief that no contestants would be able to solve this problem. However, it was eventually decided to include this problem, partly on the grounds that it is an exceptionally beautiful problem. In the event, five contestants attained perfect scores on this problem. Two of the contestants found an ingenious and short solution, which had eluded the combined efforts over several days of the 93 jury members and the problem selection committee. 


\section{Contest and Coordination}

The Irish contestants travelled to Vietnam on July 23 and attended the official opening ceremony the following day. They were accompanied by the deputy leader, Dr. Gordon Lessells. The contest was scheduled to take place in Hanoi on July 25 and 26. A total of 520 contestants from 93 countries took part, making this the largest IMO yet.

At the IMO, marking is called coordination and is a very rigorous procedure that takes place during the two days immediately following the contest. Extremely detailed marking schemes are prepared for each problem and students papers are marked independently by their own delegation (leader, deputy and observer) and by the local organisers. These two groups then meet and agree on an integer mark between 0 and 7 for each attempted solution. Thus a perfect score in the IMO is 42 (a rare and noteworthy achievement).

This year the Irish contestants performed exceptionally well. The following table details the score achieved by each contestant on each problem.

\begin{tabular}{l|ccccccc} 
& P1 & P2 & P3 & P4 & P5 & P6 & Total \\
\hline Stephen Dolan & 7 & 0 & 0 & 7 & 0 & 0 & 14 \\
Galin Ganchev & 0 & 2 & 0 & 7 & 1 & 0 & 10 \\
Derek O'Brien & 2 & 1 & 0 & 1 & 0 & 0 & 4 \\
Jamie Judd & 3 & 1 & 0 & 7 & 0 & 0 & 11 \\
Yuecong Wang & 0 & 1 & 0 & 7 & 0 & 0 & 8 \\
Katherine McCutcheon & 0 & 1 & 0 & 3 & 0 & 0 & 4
\end{tabular}

The bronze medal cutoff was 14 or higher. Accordingly, Stephen Dolan was awarded a bronze medal. Any student who does not achieve medal status, but who has recorded a perfect score on a problem is awarded an honourable mention. Thus, Galin Ganchev, Jamie Judd and Yuecong Wang obtained honourable mentions. Stephen is only the sixth Irish contestant to win a medal at the IMO. The total score of 51 is a very creditable score for an Irish team, and Ireland ranked $63 \mathrm{rd}$ among all participating nations. This is quite good, given that we have such a small population. Full details of all scores and awards can be found the official IMO website: http://www.imo-official.org/

The closing ceremony of the 48th International Mathematical Olympiad took place in Hanoi on July 30. The Spanish delegation 
gave a presentation on the 2008 IMO which is due to take place in Madrid from July 10-22.

\section{ACKNowledgements}

Thanks are due to the mathematicians at UCD, NUIM, UL, UCC and NUIG who volunteered to run mathematical enrichment courses and problem solving courses throughout the year. These courses allow students to prepare for contests such as the IMO and the Irish Mathematical Olympiad, as well as encouraging many students to pursue mathematics at third level.

Thanks to the University of Limerick for providing facilities and accommodation for the pre IMO training camp.

Also, thanks to Doreen McMorris of the Department of Education and Science Irish Olympiad Participation Committee, for providing support and funding for the Irish team to travel to Vietnam.

Finally, thanks to our Vietnamese hosts for doing such an excellent job of organising this year's IMO.

Jim Cruickshank,

Department of Mathematics,

NUI Galway,

Galway, Ireland

james.cruickshank@nuigalway.ie

Received on 10 January 2008. 